\title{
Multilayer Mie Scattering Model for Investigation of Intracellular Structural Changes in the Nucleolus and Cytoplasm
}

\author{
S. Saltsberger, I. Steinberg, and I. Gannot \\ Department of Biomedical Engineering, Faculty of Engineering, Tel-Aviv University, Tel-Aviv 69978, Israel \\ Correspondence should be addressed to I. Gannot, gannot@eng.tau.ac.il
}

Received 15 July 2011; Accepted 11 September 2011

Academic Editor: Baohong Yuan

Copyright () 2012 S. Saltsberger et al. This is an open access article distributed under the Creative Commons Attribution License, which permits unrestricted use, distribution, and reproduction in any medium, provided the original work is properly cited.

Light scattering from biological cells has been used for many years as a diagnostic tool. Several simulation methods of the scattering process were developed in the last decades in order to understand and predict the scattering patterns. We developed an analytical model of a multilayer spherical scattering cell. Here, we describe the model and show that the results obtained within this simple method are similar to those obtained with far more complicated methods such as finite-difference time-domain (FDTD). The multilayer model is then used to study the effects of changes in the distribution of internal cell structures like mitochondria distribution or nucleus internal structures that exist in biological cells. Such changes are related with cancerous processes within the cell as well as other cell pathologies. Results show the ability to discriminate between different cell stages related to the mitochondria distributions and to internal structure of the nucleolus.

\section{Introduction}

Light scattering from biological cells has been used for many years as a tool for the measurement and characterization of cell size and cell internal structure [1]. Automatic cellular and intracellular anatomical and physiological processes characterization might assist, in the diagnosis of pathological conditions and in controlling the effectiveness of a given therapy. In order to use light scattering to measure cellular level parameters and hence detect possible pathologies, one has to record the scattered signals, one cell at a time, and use that information to analyze the cell state.

One application in which a better model of light scattering from a single cell can be used to improve cell characterization in flow cytometry is presented in [2]. Here, flowing cells are individually probed by light and are characterized and sorted based on their scattering and/or fluorescence properties. In light scattering-based flow cytometry applications, two critical factors measured are the fractions of forwardscattered and side-scattered light (typically near 90 degrees), which are used to assess cell size and shape, respectively [2]. By plotting the forward scattered light against the side scattered light for a large number of of individual cells, one can then apply statistical methods to identify various cell popu- lations present inside of a cell suspension. Thus, any method that improves our understanding of how different structural compositions inside of single cells affect the scatter pattern can potentially have an enormous impact in applications where flow cytometry is used.

The general conclusions from the above studies about the light scattering dependence on the cell properties can be summarized as follows: gross cell size dominates the forward scattering (near zero angles) via diffraction processes. The nucleus is responsible for the scattering at slightly larger angles. Small organelles are responsible for the scattering at even larger angles, while light scattering in the backward direction (near 180 degrees) is mostly due to large subcellular structures [3].

It is clear that one needs a thorough understanding and the ability to characterize the processes and the patterns involved in the scattering of electromagnetic radiation (e.g., laser or LED light) from cells. Detailed simulation techniques are invaluable in applying cellular light scattering analysis.

To describe the scattering process in the optical domain (typically $0.4-1.1 \mu \mathrm{m}$ ), where the size of the investigated cells or subcellular organelles is comparable and sometimes smaller than that of the wavelength, one needs to use a full electromagnetic wave scattering theory like Mie theory. 
Conceptually simpler approximations like those of Rayleigh or geometrical optics approximation cannot be applied in all of the cases [4-7].

Over the last decades, large efforts were devoted by many researchers to find theoretical and computational methods that can be used for a simulation of the scattering process of light by cells and intracellular organelles. These methods can be divided generally to analytical and numerical methods.

Analytical methods solve Maxwell's equations for an object with a given refractive index and a simple shape (e.g., a sphere, cylinder [4], or other shape [8]). The complete analytical solution of Maxwell's equations for homogenous spherical object was published by Mie at the beginning of the 20th century. More recent works used a coated sphere model to describe the cell, which consists of two spherical layers. The internal layer describes the cell's nucleus, and the outer layer describes the cytoplasm of the cell $[1,9]$. Recently, more complex variations of Mie-like analytical models of the cell, that use multilayer configurations, were proposed and described in general terms $[4,10-12]$ and were used in a best fitting scheme between the experimental scattering pattern of blood cells and the theoretical scattering calculations in flow cytometry [13].

Numerical methods use a finite, multielement space/time grid to convert Maxwell's equations to a finite elements/differences set of equations in space and time. In the last decade, the FDTD (finite difference time domain) numerical method [14-16] has been used by several research groups in order to simulate the light scattering pattern from biological cells $[17,18]$. Recent example is the work of Tanev et al. who used FDTD to simulate the interaction of light with cellular structures [19] and applications for flow cell cytometry [20]. The discrete set of equations can be solved using numerical methods, to give the resulting distribution of electromagnetic fields in the cell and in its close vicinity. One then can get the electromagnetic field distribution in the far-field using "near to far field transformation" [16]. The main advantage of the FDTD and other numerical methods is the ability to simulate cells of arbitrary structures and not just "idealized" geometrical shapes.

However, numerical solutions might consume a large amount of computing resources because of the large number of grid elements that need to be handled even in a moderately complex problem. Moreover, in most cases one only has a simplified and idealized knowledge on the cell shape and internal structure.

In this paper, we are describing a multilayer Mie-like model that was developed to study the effects of spherically symmetric refractive index distributions on the scattering patterns of light scattered from a single cell. We show that the predictions of such model are similar to results of an FDTD model. Then, we investigate different mitochondria distributions in the cell. It was shown that cancerous processes are responsible for changes in the mitochondria distributions, and therefore, detection of such changes is of great interest. We also study the effect of internucleic structures. We demonstrate how high and low spatial frequency (hence detailed fine structure) fluctuations in the refractive index of the nucleus affect the light scattering.

\section{Models and Methods}

2.1. The Multilayer Sphere Model. The basic multilayer sphere model for electromagnetic scattering was described in the literature long ago $[4,21]$. Enhancements to the model and to the computational algorithms were described later $[10,11,22,23]$. The basic model is depicted in Figure 1. We are using the notation of Kerker [4].

In Figure 1,

$$
\alpha_{i}=\frac{2 \pi r_{i}}{\lambda_{i}}, \quad 1 \leq i \leq L
$$

is the Mie size parameter of the $i$ th spherical shell which has a radius $r_{i}$ measured from the cell center. $\lambda_{0}$ is the wavelength of the radiation in the external medium (with index $m_{0}$ ). $m_{i}$ is the (possibly complex) refractive index of the respective layer.

Obtained from the multilayer Mie model are the Mie scattering coefficients $a_{n}$ and $b_{n}$ which are equivalent to, but somewhat more complicated from, the coefficients in the case of a homogeneous sphere (one-layer model) [4]. The index $n$ is used in summations of Mie coefficients as will be outlined below. The coefficients $a_{n}$ and $b_{n}$ are given as a ratio of two determinants.

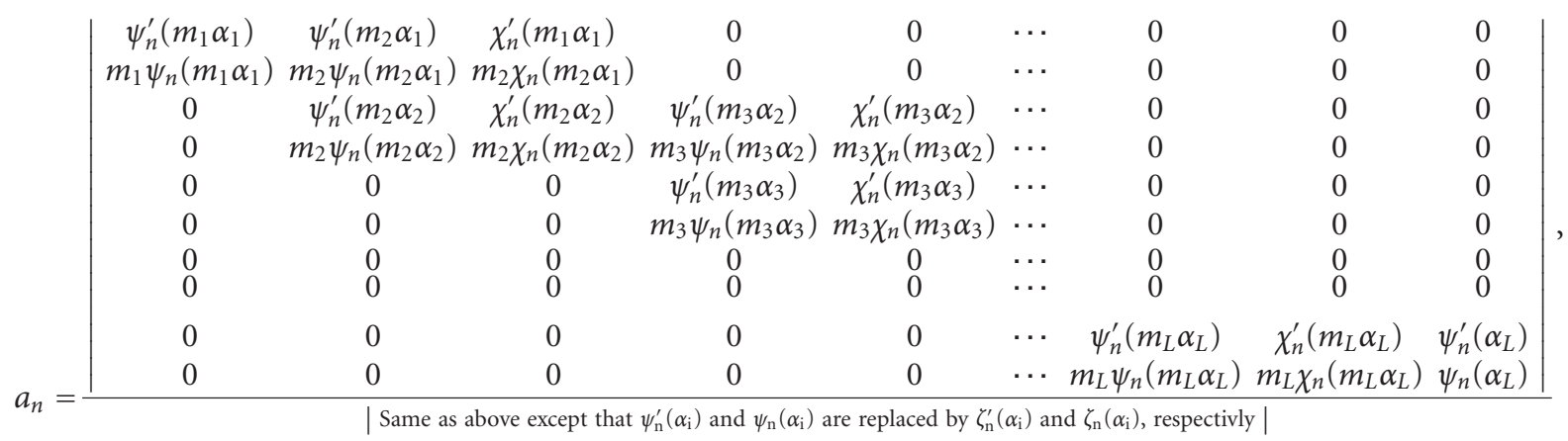




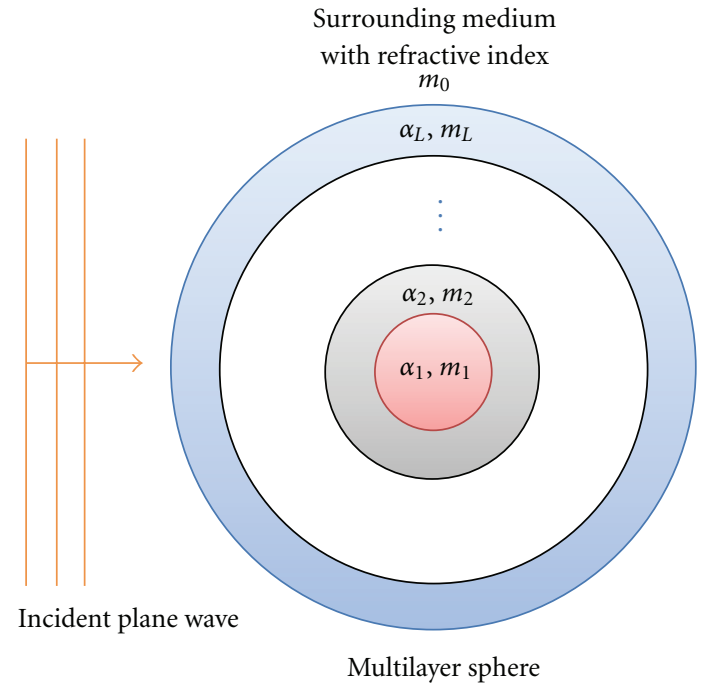

FIgURE 1: Multilayer sphere model with $L$ layers and zero indexed environment parameters. A plane electromagnetic wave is coming from the left.

where primes denote the derivative of the respective function and the various functions in (2) are outlined below [4, 24, 25]

$$
\begin{gathered}
\psi_{n}(x)=\sqrt{0.5 \pi x} J_{n+1 / 2}(x), \\
\chi_{n}(x)=-\sqrt{0.5 \pi x} N_{n+1 / 2}(x) .
\end{gathered}
$$

These are the Ricatti-Bessel functions of the first and second kind, respectively, and $J_{n+1 / 2}(x)$ and $N_{n+1 / 2}(x)$ are the half integral-order Bessel functions of the first and the second kinds (the second kind function called also Neumann function).

The function $\zeta_{n}(x)$ is the Ricatti-Bessel function of the third kind defined by

$$
\zeta_{n}(x)=\psi_{n}(x)+i \chi_{n}(x)
$$

The solution for $b_{n}$ can be obtained from that of $a_{n}$ by raising the " $m$ 's" from the even numbered rows of the determinants to the odd numbered rows immediately above. The actual dimensions of the determinants depend on the number of layers in the model and are given by

$$
\operatorname{dim}=2 L,
$$

where $L$ is the number of layers. We investigated a model which contains up to 4 layers, which gives us determinants with dimensions of $8 \times 8$.

Knowing $a_{n}$ and $b_{n}$, we can calculate the amplitude of the scattered electromagnetic waves in a given scattering angle $\theta$ and in any of the two possible polarizations: parallel and perpendicular

$$
\begin{aligned}
& S_{1}(\alpha, m, \theta) \\
& =\sum_{n=1}^{\infty} \frac{2 n+1}{n(n+1)}\left\{a_{n}(\alpha, m) \pi_{n}(\cos \theta)+b_{n}(\alpha, m) \tau_{n}(\cos \theta)\right\}, \\
& S_{2}(\alpha, m, \theta) \\
& \quad=\sum_{n=1}^{\infty} \frac{2 n+1}{n(n+1)}\left\{a_{n}(\alpha, m) \tau_{n}(\cos \theta)+b_{n}(\alpha, m) \pi_{n}(\cos \theta)\right\} .
\end{aligned}
$$

In the case of multilayer model, the Mie size parameter and the complex refractive index are vectors with one component for every layer. The angular functions $\pi_{n}$ and $\tau_{n}$ are defined by

$$
\begin{gathered}
\pi_{n}(\cos \theta)=\frac{P_{n}^{(1)}(\cos \theta)}{\sin \theta}, \\
\tau_{n}(\cos \theta)=\frac{d}{d \theta} P_{n}^{(1)}(\cos \theta),
\end{gathered}
$$

where

$$
P_{n}^{(1)}(x)=\frac{1}{2^{n} n !}\left(1-x^{2}\right)^{1 / 2} \frac{d^{n+1}}{d x^{n+1}}\left(x^{2}-1\right)^{n}
$$

is the associated Legendre polynomial of order $n$ and degree 1 [4].

The corresponding measurable intensity functions can be obtained from the amplitudes (6) by

$$
\begin{aligned}
& i_{1}(\alpha, m, \theta)=\left|S_{1}(\alpha, m, \theta)\right|^{2}, \\
& i_{2}(\alpha, m, \theta)=\left|S_{2}(\alpha, m, \theta)\right|^{2} .
\end{aligned}
$$

In a computer code, an important consideration to be made is the number of terms $n_{c}$ to be taken for the amplitude series (6) in order to preserve the required numerical precision. A relatively simple criterion for the number of terms exist in the literature [26-28]

$$
n_{c}=\operatorname{Round}\left(\alpha+4.05 \alpha^{1 / 3}+2\right) \text {, }
$$

where the Round function means rounding to the nearest integer. It can be seen by a simple calculation that the size parameter $\alpha$ for a typical case of biological cell with a radius of $5 \mu \mathrm{m}$ and the wavelength of $0.5 \mu \mathrm{m}$ is approximately 63 . Therefore, the number of terms required is 81 .

2.2. Mitochondria and Nucleus Refractive Index Fluctuations Distribution in the Multilayer Model. In order to study the mitochondrial distribution effect on the light scattering pattern of the cell, we divided the cell interior to 4 parts or zones as depicted in Figure 2: the nucleus (1), the perinuclear zone (2), the mid zone (3), and the membrane zone (4). The surrounding media is composed Extra Cellular Fluid (ECF). 


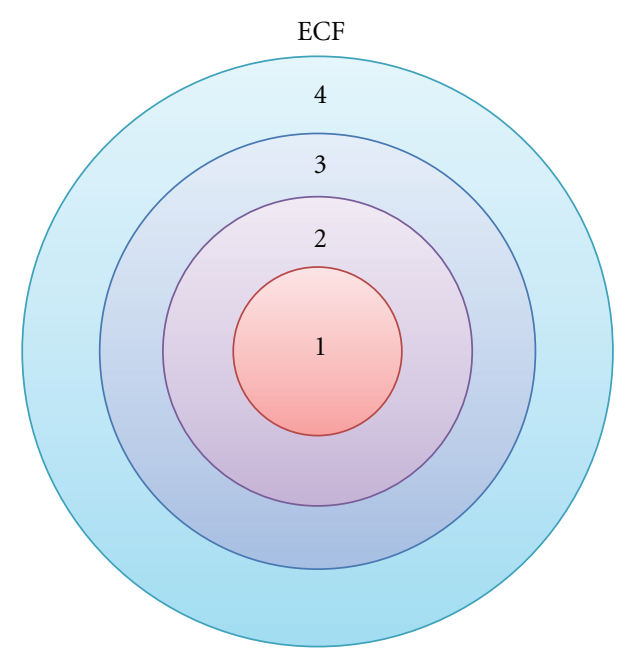

Figure 2: 4 layer sphere model: (1) Nucleus, (2) Perinuclear zone, (3) Mid zone (4) membrane zone. The surrounding is Extra Cellular Fluid (ECF).

We assume that the mitochondria distribution is represented by an equivalent distribution of refractive index in the cytoplasm region of the cell. Different cell types or states manifest themselves in the existence of certain amount of mitochondria in one of the cytoplasm zones defined above. The radii of the nucleus and of the membrane zone (i.e., whole cell's radius) are taken from the literature [17] as well as the refractive indices of the various parts of the cell and its environment. Since we divided the distance from the nucleus membrane to the cell membrane into three equal parts, the radii of the perinuclear zone and the mid zone are also determined.

The equivalent refractive index is the volume average of the cytoplasm and the mitochondria refractive indices in a certain zone. In order to calculate the volume averaged refracted index, we used the following formulas:

$$
\begin{gathered}
m_{\mathrm{avr}, i}=v_{\mathrm{mito}, i} m_{\text {mito }}+v_{\mathrm{cyto}, i} m_{\mathrm{cyto}} \\
v_{\mathrm{mito}, i}=\frac{1}{r_{i}^{3}-r_{i-1}^{3}} \sum_{j=1}^{N_{i}} \rho_{\text {mito }, j}^{3} \\
v_{\mathrm{cyto}, i}=1-v_{\text {mito }, i}
\end{gathered}
$$

where, $i$ : zone index, that can takes one of the values 2,3 or 4 for perinuclear, mid and membrane zone respectively; $m_{\text {mito }}, m_{\text {cyto }}$ : refractive index of the mitochondria and the cytoplasm respectively; $v_{\text {mito }, i}, v_{\text {cyto }, i}:$ volume fraction of mitochondria and cytoplasm respectively in zone $i ; m_{\mathrm{avr}, i}$ : average (effective) refractive index of the zone $i ; r_{i}$ : radius of the zone $i$, measured from cell's center; $\rho_{\text {mito, } j}$ : radius of the $j$ th mitochondrion (in the zone $i$ ); $N_{i}$ : number of mitochondrion in the zone $i$.

In (11) above, the total mitochondria volume can be calculated as the sum of all the single mitochondrion volumes in the respective zone $i$. Every mitochondrion is modeled as a sphere with radius $\rho_{\text {mito,j }}$.

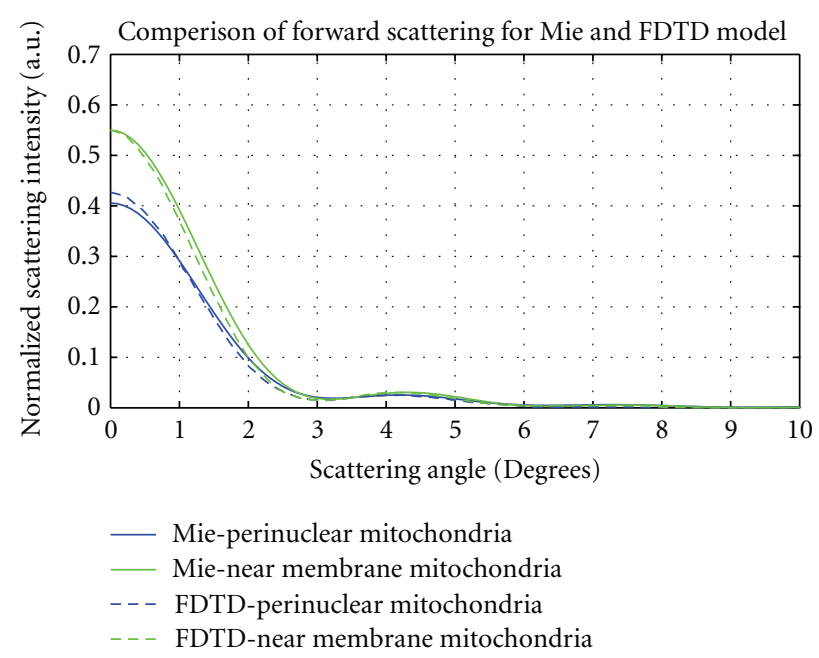

FIGURE 3: Forward scattering intensity as a function of scattering angle. Full lines: analytic multilayer Mie model. Dashed lines: Numerical FDTD model from Figure 2(a) of Su et al. [18] showing similar behavior. Both perinuclear mitochondria (blue) and near membrane mitochondria cases (red) are shown.

Changes in the nucleus might result in coarser or fine structures of the refractive index at the nucleolus compared to a normal cell. This means lower or higher spatial frequency content of the refractive index fluctuation in the nucleolus. In the multilayer model it is natural to approximate the nucleus refractive index fluctuations as a nucleus with $N$ spherical shells with spatial frequency of $0.5 N r_{\text {nuc }}^{-1}$ cycles $/ \mu \mathrm{m}$, where $r_{\text {nuc }}$ is the radius of the nucleus in microns. The refractive index of odd shells is $m_{\mathrm{avr}}-\Delta m$ and of even shells is $m_{\mathrm{avr}}+$ $\Delta m$ where $\Delta m$ is relatively small amount.

\section{Results}

3.1. Comparison with FDTD Model. The details of the near forward scattering (0-10 degrees) pattern of the average polarization are shown in Figure 3 below. For comparison, the data from Figure 2(a) of Su et al. [18], which is a result of FDTD computation for a similar geometry, is plotted in dashed lines. Refractive indices and radii were taken to match $\mathrm{Su}$ et al. [18]. However, randomization of the mitochondria locations was neglected and replaced with spatial averaging as discussed in Section 2.2. The results of both calculations are very similar for both perinuclear mitochondria and near membrane mitochondria.

3.2. Mitochondria Distribution Effects. For further investigation of the effects of different mitochondria distributions, the multilayer model was run with the following parameters. These parameters are taken from previous publications [17, 18 ] and their values are summarized in Table 1 . The radii of zones 2, 3, 4 in the cytoplasm (see Figure 2 above) were taken to be equal.

Based on these parameters, the scattering intensities functions $i_{1}$ and $i_{2}$ (parallel and perpendicular polarizations resp.) were plotted. Figures $4(\mathrm{a})$ and $4(\mathrm{~b})$ show the intensities 


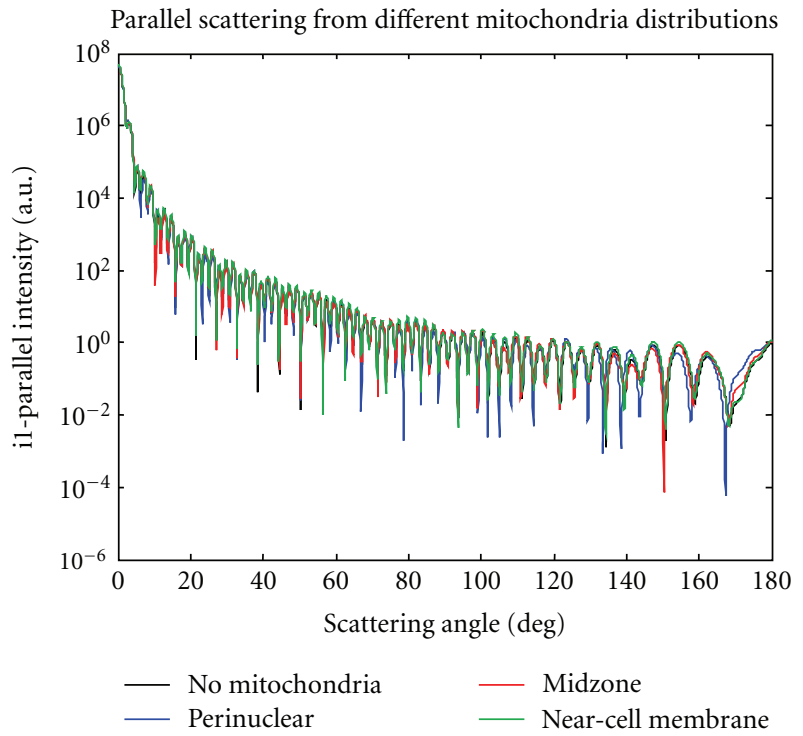

(a)

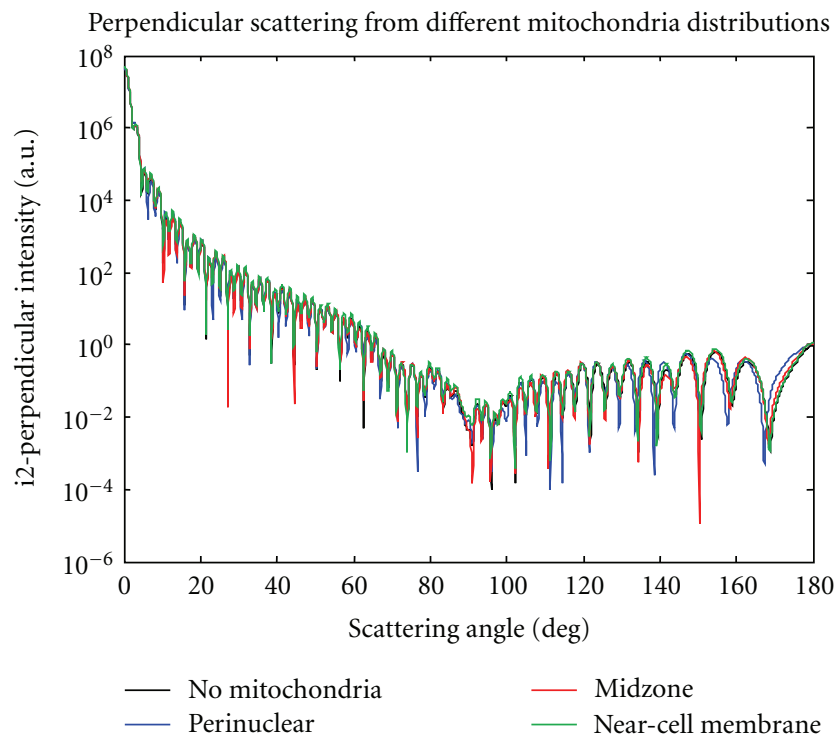

(b)

FIGURE 4: Scattered intensities as a function of scattering angles for different mitochondria distributions. (a) $i_{1}$ parallel polarization, (b) $i_{2}$ perpendicular polarization. The different colors represent different mitochondria distributions.

TABLE 1: Summary of simulation parameters.

\begin{tabular}{lccc}
\hline Item & Value & Units & Comments \\
\hline $\begin{array}{l}\text { Number of } \\
\text { mitochondria }\end{array}$ & 180 & No. & In the cytoplasm \\
$\begin{array}{l}\text { Mitochondrion radius } \\
\text { Laser wavelength }\end{array}$ & 0.35 & $\mu \mathrm{m}$ & \\
$\begin{array}{l}\text { Cell's radius } \\
\text { Nucleus radius }\end{array}$ & 7.488 & $\mu \mathrm{m}$ & For argon laser \\
$\begin{array}{l}\text { Extracellular fluid's } \\
\text { index } \\
\text { of refraction }\end{array}$ & 1.50 & $\mu \mathrm{m}$ & \\
$\begin{array}{l}\text { Cytoplasm's index } \\
\text { of refraction }\end{array}$ & 1.37 & No. & $\begin{array}{c}\text { Relative to } \\
\text { vacuum }\end{array}$ \\
$\begin{array}{l}\text { Mitochondria's index } \\
\text { of refraction }\end{array}$ & 1.42 & No. & $\begin{array}{c}\text { Relative to } \\
\text { vacuum } \\
\text { vacuum }\end{array}$ \\
$\begin{array}{l}\text { Nucleus' index of } \\
\text { refraction }\end{array}$ & 1.39 & No. & $\begin{array}{c}\text { Relative to } \\
\text { vacuum }\end{array}$ \\
\hline
\end{tabular}

as a function of the scattering angle (forward is a zero scattering angle) for a few mitochondria distribution of interest. Due to the exponentially decaying nature of the intensities, the magnitude is shown in logarithmic scale in order to see the behavior in large scattering angles.

Since the overall differences between cases are small, we focus on side-scattering which is related to cell structure. Figure 5 zooms into this region of interest to show the differences in the $i_{2}$ side-scattering between mitochondria distributions. Here mitochondria positions were randomized ten times for each distribution to see the variance of theses different situations. One can deuce from the figure that the further the mitochondria displaced from the nucleus the stronger is the side scattering. Similar results can be obtained for the $i_{1}$ intensity.

Finally, as mentioned before, it is a common practice in cell cytometry to plot side scattering intensity versus forward scattering intensity in order to cluster different cell types. This allows the application of a wide array of classifiers and clustering algorithms. Therefore, we plotted the results from Figures 4 and 5 in such manner for the different mitochondria distributions. The result is presented in Figure 6 below.

3.3. Nuclear Fine Structure Effects. In order to study the effects of the nuclear fine structures on the light scattering pattern we used the multilayer model with several spatial frequencies in the nucleus.

The results for homogenous nucleus and nucleus with 25 , 37,50 layers of refractive index modulation (spatial frequency of 5, 7.5, 10 cycles/micrometer) are depicted in Figures 7(a), 7(b) and 7(c) below.

We observe major differences between homogenous nucleolus and multilayered nucleus. Similar results are obtained for the $i_{2}$ scattering intensity.

\section{Discussion and Conclusions}

We have developed and implemented a MATLAB multilayer Mie-like model for the scattering of light from biological cells that have internal structures with spherical symmetry. The multilayer Mie-like model can be used for exvivo and invivo simulation of light scattering from biological cells.

The model is somewhat simpler than the complex FiniteDifference Time-Domain (FDTD) cell models that were recently published and used by several groups [14-18, 29]. We have showed in Figure 3 that the multilayer model gives 


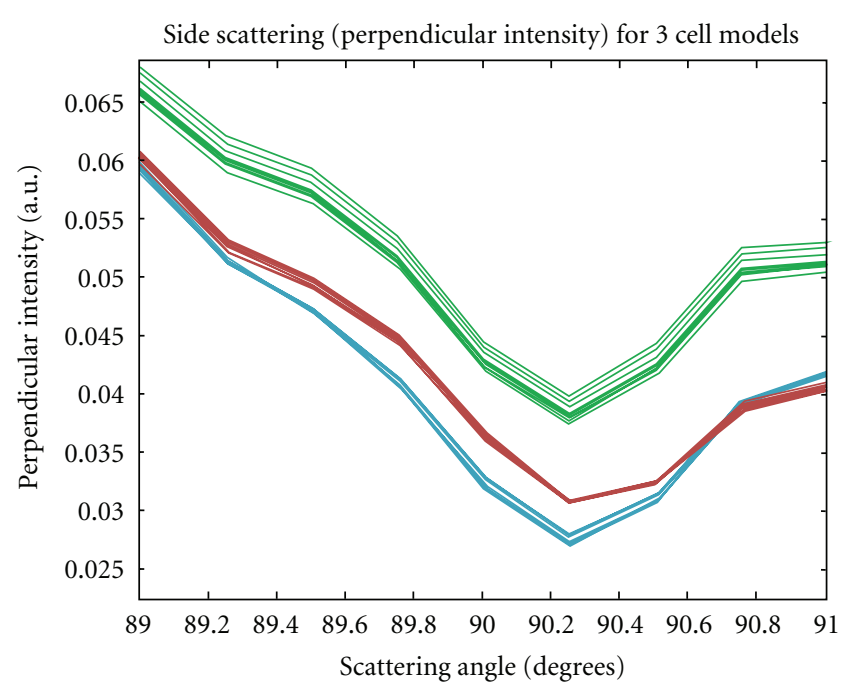

— Perinuclear mitochondria _ Near-membrane mitochondria - Midzone mitochondria

FIGURE 5: Side-scattering intensity $i_{2}$ for different mitochondria distributions. For each different distribution (represented by different color) 10 realizations of the mitochondria positions were randomized to show the variance in scattering intensity.

similar results as the FDTD model for cases having spherical symmetry in the refractive index distribution in the cell. For both perinuclear distributed and near-membrane distributed mitochondria, there is a very reasonable equivalence between the simplified analytical multilayer Mie model and the more complicated FDTD model in case of spherical symmetry.

The effect of the mitochondria distribution and quantity on the cell's light scattering pattern is an example to a cellular-based optical diagnosis method [18]. It has been shown that mitochondrial aggregation near the nuclear periphery will occur in normal cells in preparation for mitosis, while in the rest of the normal cell's life the mitochondria is distributed randomly in the cytoplasm. It also has been observed that mitochondrial hyperplasia can be encountered in tumors from different organs, (also called oncocytomas) [30]. In Figure 4, which plots the scattering patterns of the 3 cell types on a log scale, the scatter patterns of the 3 cell types appear similar. However, when the data is plotted on a linear scale, differences between normal (perinuclear mitochondria), precancerous (midzone mitochondria), and cancerous (near-membrane mitochondria) cells begin to emerge, mostly for the side scattering intensity (shown in Figure 5), with the fraction of scattered light being roughly $30 \%$ higher in cancerous cells than normal or precancerous cells. The forward scattering component is slightly less sensitive to the location of mitochondria.

These results are consistent with earlier findings in flow cytometry that show that the side scatter component is more sensitive to cell structure, while the forward scatter component is more sensitive to cell size [2]. Moreover, plotting the simulation output on a scatter plot, as is frequently done in flow cytometry data, reveals that the 3 cell groups can be easily classified. The ability to assess intracellular structures

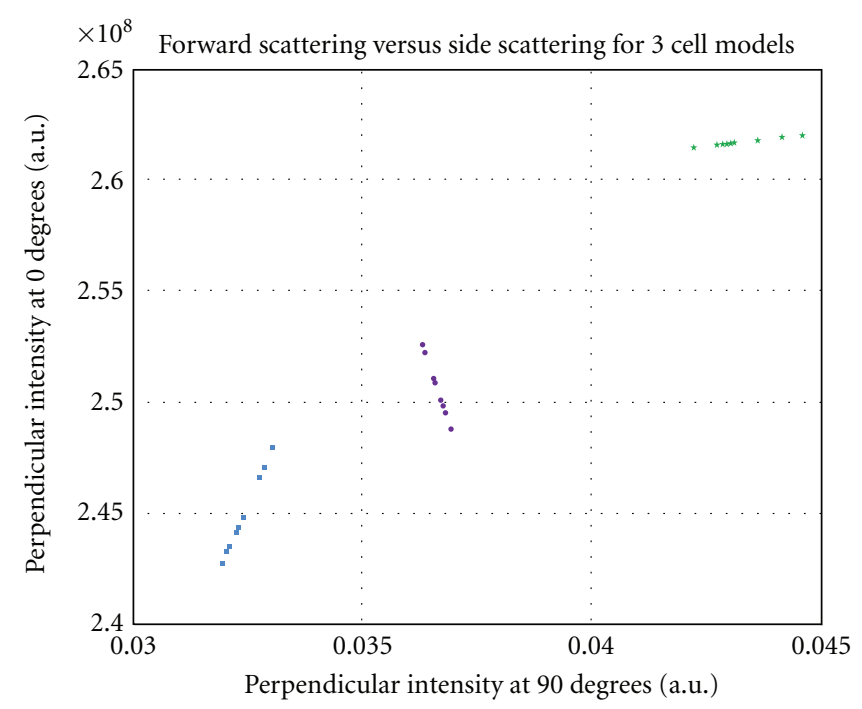

- Perinuclear mitochondria * Near-membrane mitochondria

- Midzone mitochondria

FIGURE 6: Scatter plot of the $i_{2}$ forward scattering intensity versus the $i_{2}$ side scattering intensity for the 10 realizations of each of the 3 different mitochondria distributions. This plot clearly shows the differentiation between the cases and spread of each distribution.

such as location of mitochondria using flow cytometry can add a new dimension to this well-established method.

Although the model is based on concentric spherical layers, the actual shape of cells frequently deviates from this geometry. If the cells had a more oblong, ellipsoidal shape (with the long axis perpendicular to the incoming incident wave), we suspect that the scatter patterns would deviate from Figures 4-6 in that there would be less light scattered in the $90^{\circ}$ direction, while there would be more light scattered in the forward direction. Alternatively, if the cells had long dendritic-like processes, we expect higher intensity in the $90^{\circ}$ direction and less light in the forward direction. Of note, many cancers originate in epithelial tissue that has more spherical/ellipsoidal shape than a dendritic shape [31].

While noncancerous cells often exhibit round or otherwise ordered shape, cancerous cells often appear with irregular shapes. The lack of symmetry of such cells prevents simple analytical modeling, enforcing regression to the more complex FDTD models. In such cases, the importance of the analytical Mie model is double. First, as explained in the introduction, scattering at large angles is related to subcellular organs and unrelated to the overall shape of the cell. Thus, the Mie model described here is a fair estimate of the scattering at large angles even for irregular-shaped cells. Second, since each cell is measured with respect to a random frame of reference, the average scattering from a population of irregular-shaped cells is similar to that of a hypothetical spherical cell described by the Mie model presented here.

Another example to a possible diagnostic feature is the nonuniformity of the refractive index in the cell nucleus. It is known that the refractive index in a certain point in the nucleus (and also in the cell) is a function of several factors, 


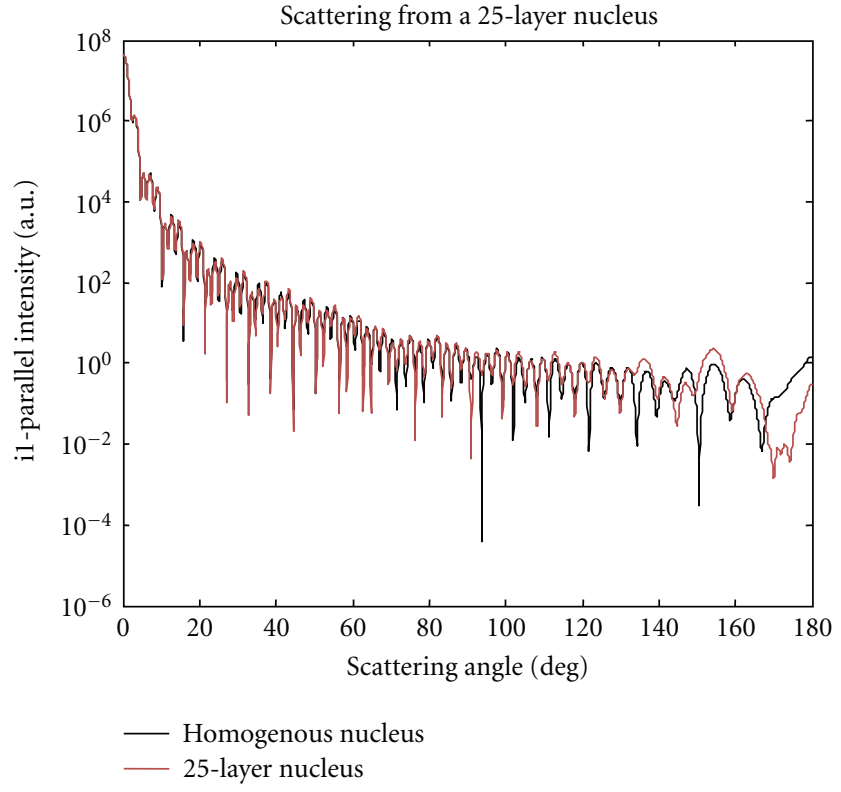

(a)

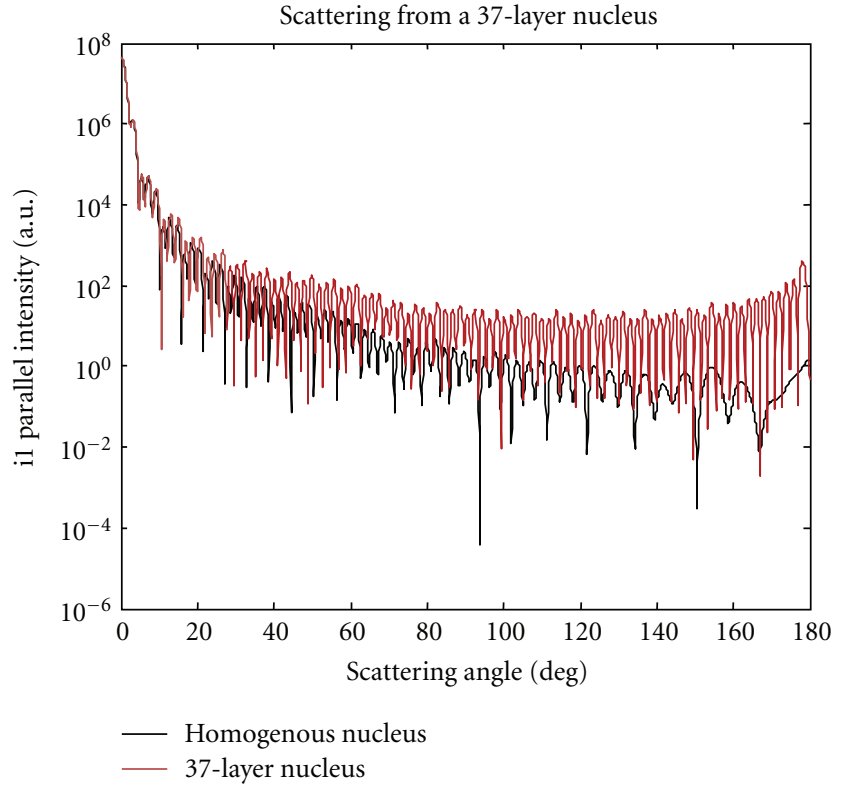

(b)

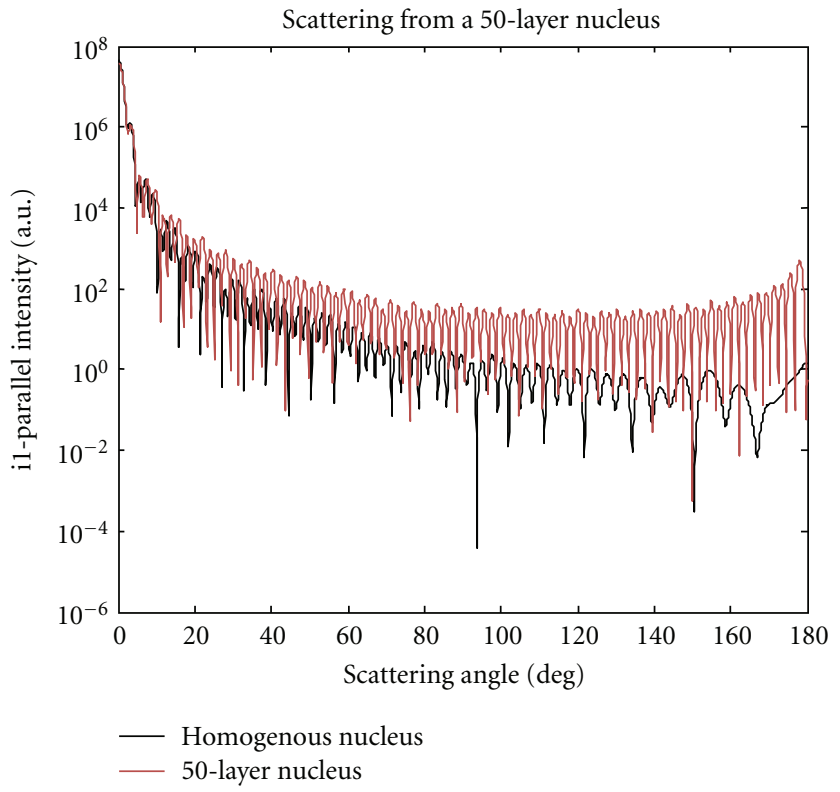

(c)

Figure 7: (a) Parallel component of the scattered intensity $i_{1}$ as a function of the scattering angle for a nucleus with refractive index fluctuations (layers) of 5 cycles/micron spatial frequency. (b) Parallel component of the scattered intensity $i_{1}$ as a function of the scattering angle for a nucleus with refractive index fluctuations of 7.5 cycles/micron spatial frequency. (c) Parallel component of the scattered intensity $i_{1}$ as a function of the scattering angle for a nucleus with refractive index fluctuations of 10 cycles per micron spatial frequency.

including protein concentration [32]. As the cell goes through its life cycle, the protein concentration in the nucleus and hence the refractive index distribution is changing because of the preparation to and the beginning of the cell mitosis [33]. Nucleus refractive index nonuniformity effects on the light scattering pattern had been recently investigated using FDTD methods [17]. The main result was that higher frequency fluctuation in the nucleus refractive index results in more scattering in the angles between 50 and 160 degrees.
It was also shown in the literature [34] that the nucleus of cancer cells depicts internal structural changes, compared with normal cells. These changes are specific to the cancer type and can assist in diagnosis and staging.

Applying the multilayer Mie model, we can see that in general, the scattering becomes higher as the spatial frequency of the refractive index variation is higher. This is true for high scattering angle, not in the forward direction but from 10 to 20 degrees. This result is in general agreement 
with Drezek et al. [17] that used the FDTD method for their simulation, although that in their simulation they used refractive index patterns in the cytoplasm rather than in the nucleus.

Another interesting feature that we see is the regular peaks pattern that becomes visible for higher spatial frequencies. This pattern is not present in the FDTD simulation [17]. The difference can be explained by the fact that we used very regular periodic spherically symmetric structure of refractive index distribution. The periodic structure is in fact like a $3 \mathrm{D}$ spatial diffraction grating, giving rise to many resonance peaks in the diffraction pattern. The FDTD simulation [17], on the other hand, used a non-periodic patterns like certain number of organelles of specific size and refractive index at specific locations or randomly generated refractive index patterns with a certain range and frequency of fluctuations. The randomness nature of the patterns they used was acted as an averaging component that prevents the creation of resonant peaks because of the random phase of the interference. The resulting scattering pattern is much less coherent as in our regular periodic case.

In the future, we intend to check its potential use in an algorithm for a cell classification system. A single-cell classification algorithms are used routinely, for example, in a cell cytometry equipment [2]. The behavior near the forward direction probably can be used as a discriminating feature in an automatic cell classifier.

Another potential application that can use this model is the calculation of the invivo back-scattering from a tissue bulk that composed of many cells, some of them depict a pathology in a certain level. The purpose of the calculations in this case is to enable a classification of invivo intracell level pathologies or the staging of pathology as a part of a therapy. In this case, one needs to compute the aggregate effect of the scattering from many cells and also incorporate the tissue matrix and the (possibly variable) blood effect on the backscattering.

\section{References}

[1] R. A. Meyer and A. Brunsting, "Light scattering from nucleated biological cells," Biophysical Journal, vol. 15, no. 3, pp. 191-203, 1975.

[2] H. M. Shapiro, Practical Flow Cytometry, John Wiley \& Sons, New York, NY, USA, 4th edition, 2003.

[3] C. Liu, C. Capjack, and W. Rozmus, "3-D simulation of light scattering from biological cells and cell differentiation," Journal of Biomedical Optics, vol. 10, no. 1, pp. 014007.1014007.12, 2005.

[4] M. Kerker, The Scattering of Light and other Electromagnetic Radiation, Academic Press, 1969.

[5] H. C. van-de-Hulst, Light Scattering by Small Particles, Dover, New York, NY, USA, 1981.

[6] C. F. Bohren and D. R. Huffman, Absorption and Scattering of Light by Small Particles, John Wiley \& Sons, New York, NY, USA, 1983.

[7] E. J. McCartney, Optics of the Atmosphere, John Wiley \& Sons, New York, NY, USA, 1976.
[8] M. I. Mishchenko, J. W. Hovenier, and L. D. Travis, Light Scattering by Nonspherical Particles: Theory, Measurements, and Applications, Academic press, 2000.

[9] A. Brunsting and P. F. Mullaney, "Light scattering from coated spheres: model for biological cells," Applied Optics, vol. 11, no. 3, pp. 675-680, 1972.

[10] R. Bhandari, "Scattering coefficients for a multilayered sphere: analytic expressions and algorithms," Applied Optics, vol. 24, no. 13, pp. 1960-1967, 1985.

[11] N. G. Volkov and V. Y. Kovach, "Scattering of light by inhomogeneous spherically symmetrical aerosol particles," Izvestya Atmosperic and Oceanic Physics, vol. 26, no. 5, pp. 381-385, 1990.

[12] L. Kai and P. Massoli, "Scattering of electromagnetic plane waves by radially inhomogeneous spheres: a finely stratified sphere model," Applied Optics, vol. 33, no. 3, pp. 501-511, 1994.

[13] A. Zharinov, P. Tarasov, A. Shvalov, K. Semyanov, D. R. van Bockstaele, and V. Maltsev, "A study of light scattering of mononuclear blood cells with scanning flow cytometry," Journal of Quantitative Spectroscopy and Radiative Transfer, vol. 102, no. 1, pp. 121-128, 2006.

[14] A. Taflove and S. C. Hagness, Computational Electrodynamics: the Finite-Difference Time-Domain Method, Artech House, 2nd edition, 2000.

[15] D. M. Sullivan, Electromagnetic Simulation Using the FDTD Method, John Wiley \& Sons, IEEE, New York, NY, USA, 2000.

[16] A. Z. Elsherbeni and V. Demir, The Finite-Difference TimeDomain Method for Electromagnetics with MATLAB Simulation, Scitech, 2009.

[17] R. Drezek, A. Dunn, and R. Richards-Kortum, "Light scattering from cells: finite-difference time-domain simulations and goniometric measurements," Applied Optics, vol. 38, no. 16, pp. 3651-3661, 1999.

[18] X. Su, K. Singh, W. Rozmus, C. Backhouse, and C. Capjack, "Light scattering characterization of mitochondrial aggregation in single cells," Optics Express, vol. 17, no. 16, pp. 1338113388, 2009.

[19] S. Tanev, W. Sun, J. Pond et al., "FDTD simulation of light interaction with cells for diagnostics and imaging in nanobiophotonics," in Handbook of Photonics for Biomedical Science, Medical physics \& biomedical engineering, CRC Press, Taylor \& Francis, 2011.

[20] S. Tanev, Wenbo Sun, J. Pond et al., "Optical imaging of cells with gold nanoparticle clusters as light scattering contrast agents: a finite difference time domain approach to the modeling of flow cytometry configurations," in Advanced Optical Flow Cytometry, pp. 35-62, 2010.

[21] P. J. Wyatt, "Scattering of electromagnetic plane waves from inhomogeneous spherically symmetric objects," Physical Review, vol. 127, no. 5, pp. 1837-1843, 1962.

[22] B. R. Johnson, "Light scattering by a multilayer sphere," Applied Optics, vol. 35, no. 18, pp. 3286-3296, 1996.

[23] A. Y. Perelman, "Scattering by particles with radially variable refractive indices," Applied Optics, vol. 35, no. 27, pp. 54525460, 1996.

[24] M. R. Spiegel, Schaum's Mathematical Handbook of Formulas and Tables, McGraw Hill, 1968.

[25] M. Abramowitz and I. A. Stegun, Handbook of Mathematical Functions, Dover, New York, NY, USA, 1972.

[26] I. K. Ludlow and J. Everitt, "Systematic behavior of the Mie scattering coefficients of spheres as a function of order," Physical Review E, vol. 53, no. 3, pp. 2909-2924, 1996. 
[27] G. Chliveros and M. A. Rodrigues, MATLAB Implementation of the Exact Solution for the n-Layer Sphere Problem, Materials \& Engineering Research Institute, Sheffield Hallam University, 2004.

[28] W. J. Wiscombe, "Improved Mie Scattering Algorithms," Applied Optics, vol. 19, no. 9, pp. 1505-1509, 1980.

[29] A. Dunn, Light Scattering Properties of Cells, University of Texas at Austin, Austin, Texas, 1997.

[30] P. L. Gourley, J. K. Hendricks, A. E. McDonald et al., "Ultrafast nanolaser flow device for detecting cancer in single cells," Biomedical Microdevices, vol. 7, no. 4, pp. 331-339, 2005.

[31] E. N. Marieb and K. Hoehn, Human Anatomy \& Physiology, Pearson Education, 2007.

[32] R. Barer and S. Joseph, "Refractometry of living cell part I: basic pronciples," Quarterly Journal of Microscopical Science, vol. 95, no. 4, pp. 399-423, 1954.

[33] A. Brunsting and P. F. Mullaney, "Differential light scattering from spherical mammalian cells," Biophysical Journal, vol. 14, no. 6, pp. 439-453, 1974.

[34] D. Zink, A. H. Fischer, and J. A. Nickerson, "Nuclear structure in cancer cells," Nature Reviews Cancer, vol. 4, no. 9, pp. 677687, 2004. 

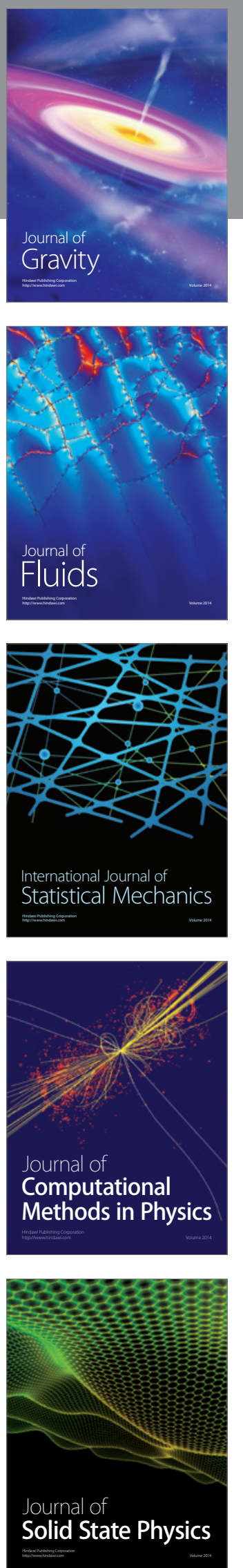

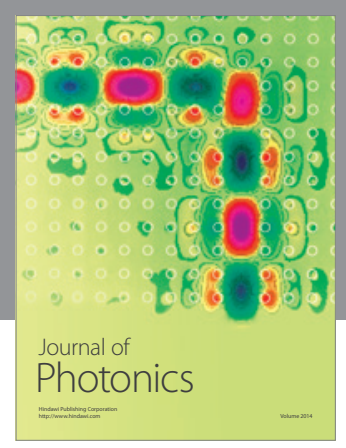

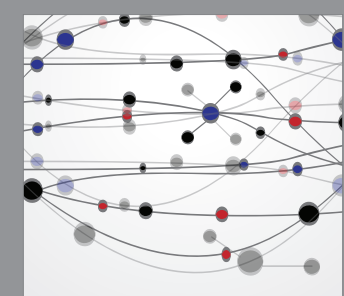

The Scientific World Journal
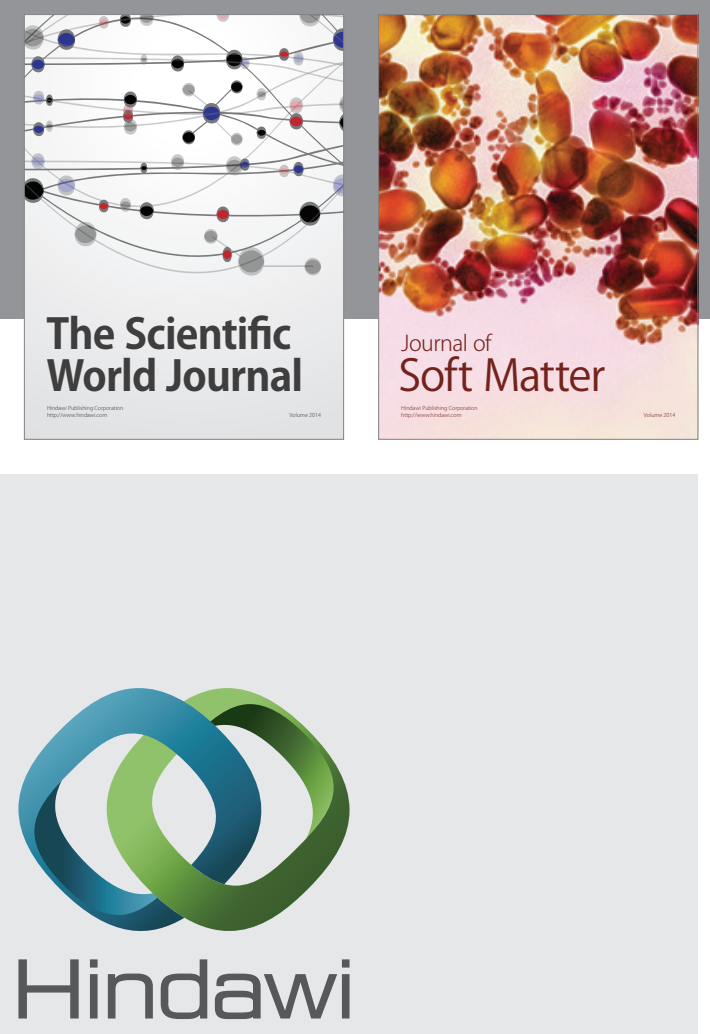

Submit your manuscripts at

http://www.hindawi.com
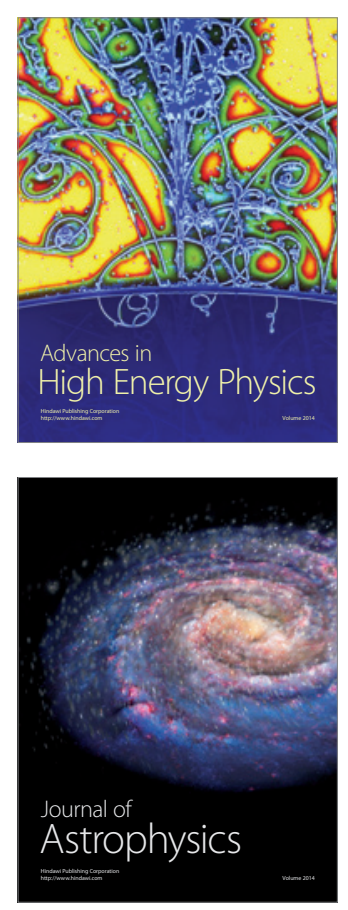
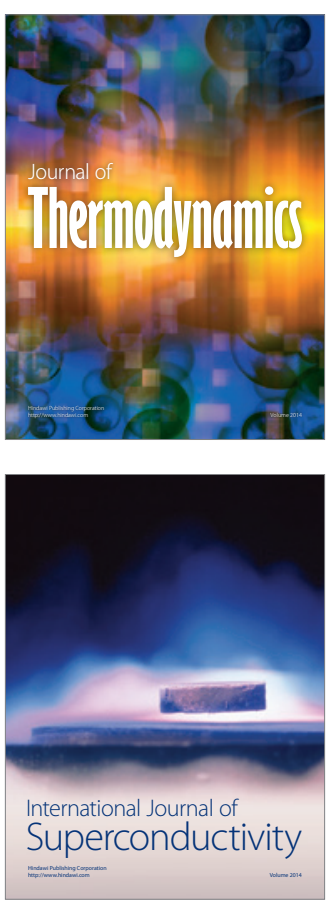
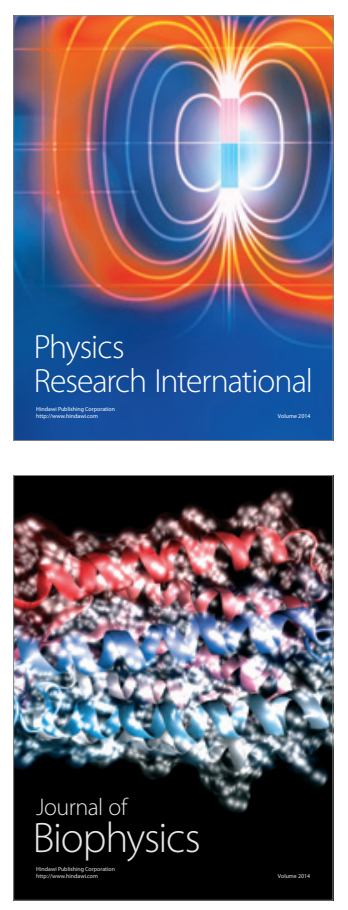
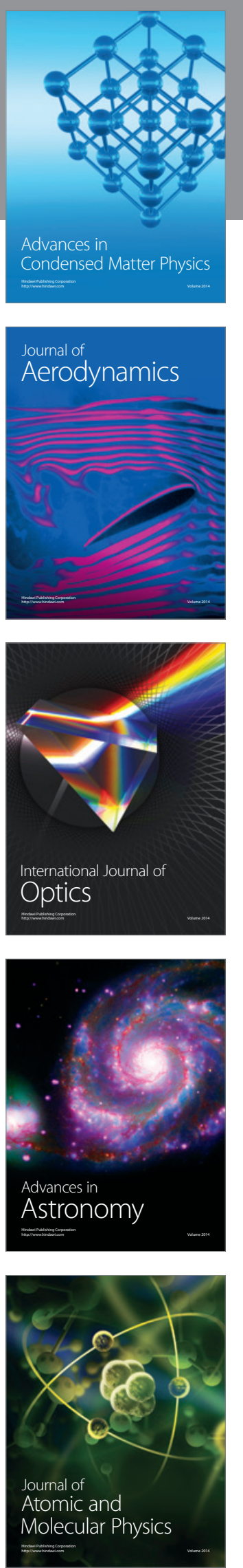